Delft University of Technology

\title{
Quantum paraelectricity probed by superconducting resonators
}

Davidovikj, D.; Manca, N.; Van Der Zant, H. S.J.; Caviglia, A. D.; Steele, G. A.

DOI

10.1103/PhysRevB.95.214513

Publication date

2017

Document Version

Final published version

Published in

Physical Review B (Condensed Matter and Materials Physics)

\section{Citation (APA)}

Davidovikj, D., Manca, N., Van Der Zant, H. S. J., Caviglia, A. D., \& Steele, G. A. (2017). Quantum paraelectricity probed by superconducting resonators. Physical Review B (Condensed Matter and Materials Physics), 95(21), [214513]. https://doi.org/10.1103/PhysRevB.95.214513

\section{Important note}

To cite this publication, please use the final published version (if applicable).

Please check the document version above.

\section{Copyright}

Other than for strictly personal use, it is not permitted to download, forward or distribute the text or part of it, without the consent of the author(s) and/or copyright holder(s), unless the work is under an open content license such as Creative Commons.

\section{Takedown policy}

Please contact us and provide details if you believe this document breaches copyrights.

We will remove access to the work immediately and investigate your claim. 


\title{
Quantum paraelectricity probed by superconducting resonators
}

\author{
D. Davidovikj, ${ }^{*}$ N. Manca ${ }^{\dagger}$ H. S. J. van der Zant, A. D. Caviglia, and G. A. Steele \\ Kavli Institute of Nanoscience, Delft University of Technology, P.O. Box 5046, 2600 GA Delft, The Netherlands
}

(Received 1 August 2016; revised manuscript received 24 May 2017; published 28 June 2017)

\begin{abstract}
Superconducting coplanar waveguide (CPW) resonators are powerful and versatile tools used in areas ranging from radiation detection to circuit quantum electrodynamics. Their potential for low intrinsic losses makes them attractive as sensitive probes of electronic properties of bulk materials and thin films. Here we use superconducting MoRe CPW resonators to investigate the high-frequency (up to $0.3 \mathrm{GHz}$ ) and low-temperature (down to $3.5 \mathrm{~K}$ ) permittivity of $\mathrm{SrTiO}_{3}$, a nonlinear dielectric on the verge of a ferroelectric transition (quantum paraelectricity). We perform a quantitative analysis of its dielectric properties as a function of external dc bias (up to $\pm 15 \mathrm{~V}$ ), rf power, and mode number and discuss our results within the framework of the most recent theoretical models. We also discuss the origin of a fatigue effect that reduces the tunability of the dielectric constant of $\mathrm{SrTiO}_{3}$, which we relate to the presence of oxygen vacancies.
\end{abstract}

DOI: 10.1103/PhysRevB.95.214513

\section{INTRODUCTION}

In recent years, coplanar waveguide (CPW) resonators have proven to be a unique tool for probing a wide variety of excitations in circuits and materials, including superconducting qubits [1], ferromagnetic spin ensembles [2], and magnons [3]. Due to their ultralow ohmic losses, they usually exhibit high quality factors, making them very sensitive to external perturbations. As their properties are, to a large extent, defined by their geometry and by the dielectric response of their environment, they make a promising candidate as probes for materials with exotic electronic properties. Transition metal oxide (TMO) heterointerfaces exhibit a variety of electronic and structural properties, including two-dimensional (2D) superconductivity [4], ferroelectric/magnetic orders [5], and negative capacitance [6]. Probably the most ubiquitous representative of transition metal oxides is $\mathrm{SrTiO}_{3}$. Its chemical stability, lattice constant, and dielectric characteristics make it one of the standard substrates for the growth of high-quality crystalline thin films.

At low temperatures $\mathrm{SrTiO}_{3}$ is characterized by an extremely high dielectric response $\left(\varepsilon_{r}=23000\right.$ below $4 \mathrm{~K}$ [7-10]) originating from an incipient ferroelectric transition (quantum paraelectricity). The permittivity is affected by electric fields, and below $4 \mathrm{~K}$ it can be lowered by more than 1 order of magnitude for a field of about $2 \mathrm{MV} / \mathrm{m}[11,12]$. The gate-tunability of the dielectric constant of $\mathrm{SrTiO}_{3}$, particularly in thin-film form, has been used in applications of resonators incorporating $\mathrm{SrTiO}_{3}$ for voltage-controlled microwave filters [13-18]. Beyond applications, one can also envision using superconducting resonators for sensing and exploring the dielectric properties of $\mathrm{SrTiO}_{3}$ itself, similar to the application of superconducting cavities to probe qubits, spin ensembles, and magnetic excitations. The integration of CPW technology with oxide heterostructures is a novel approach towards studying these complex materials as a high-precision and device-oriented technique. However, it first

\footnotetext{
*d.davidovikj@tudelft.nl

†n.manca@tudelft.nl
}

requires understanding of how the presence of the $\mathrm{SrTiO}_{3}$ substrate affects the response of planar devices working at microwave frequencies.

Here, we use superconducting CPW resonators fabricated on single-crystal $\mathrm{SrTiO}_{3}$ to explore the dielectric response of this quantum paraelectric material. As a reference, we fabricate a nominally identical resonator on single-crystal $\mathrm{Al}_{2} \mathrm{O}_{3}$ (sapphire). Since sapphire is a well-known standard substrate with low dielectric losses [19], we were able to perform a quantitative analysis on the dielectric response of the resonator fabricated on top of the $\mathrm{SrTiO}_{3}$ sample under different excitations by direct comparison of the two resonators.

\section{EXPERIMENTAL SETUP}

A superconducting coplanar waveguide resonator is fabricated on top of a $\mathrm{TiO}_{2}$-terminated single-crystal $\mathrm{SrTiO}_{3}(001)$ substrate $\left(5 \times 5 \times 0.5 \mathrm{~mm}^{3}\right)$. The CPW resonator is realized by a standard lithographic process followed by sputtering of a 135-nm-thick molybdenum-rhenium $\left(\mathrm{Mo}_{0.60} \mathrm{Re}_{0.40}\right)$ film and lift-off in acetone. A cross-sectional schematic of the device is presented in Fig. 1(a). The CPW resonator is a 45-mm-long line with a meander shape, and the central strip is $30 \mu \mathrm{m}$ wide, with a $10-\mu \mathrm{m}$ gap to the ground plane. The geometric parameters are chosen such that a significant impedance mismatch is created between the transmission line $\left(Z_{0}=50 \Omega\right)$ and the resonator $\left(Z_{C P W}=1 \Omega\right.$ at $3.5 \mathrm{~K}$, which acts as a mirror for microwave photons). This, together with the open end of the resonator, creates a $\lambda / 2$ cavity. Figure 1(b) shows a top-view optical image of the device together with a schematic of the measurement setup. Our measurement scheme provides galvanic access to the central strip, so a dc bias can be easily applied with respect to the ground plane. Reflection measurements $\left(\mathrm{S}_{11}\right)$ are performed using a vector network analyzer, while the sample is always kept at a fixed temperature of $3.5 \mathrm{~K}$.

We obtain information on the dielectric properties of the $\mathrm{SrTiO}_{3}$ by direct comparison with an identical resonator, fabricated on top of a sapphire substrate (see Supplemental Material S1 [20]). As the inductance of both waveguides is the 


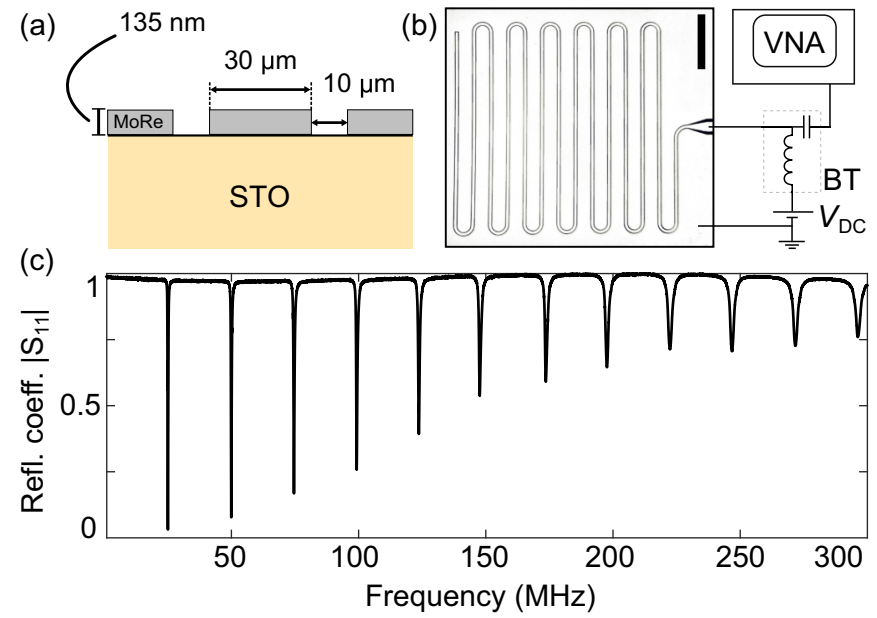

FIG. 1. Design of a superconducting CPW resonator as a probe of the dielectric response of $\mathrm{SrTiO}_{3}$. (a) Cross-sectional schematic of the device. (b) Top-view optical image of the $\lambda / 2 \mathrm{CPW}$ resonator with a schematic of the measurement setup. (VNA: vector network analyzer; BT: bias tee). (c) Reflection measurement $\left|\mathrm{S}_{11}\right|$ taken at $3.5 \mathrm{~K}$. Twelve resonance modes are visible in the $1-300-\mathrm{MHz}$ range.

same, the ratio of the frequencies of the fundamental modes of the two resonators $\left(f_{1}^{\mathrm{Al}_{2} \mathrm{O}_{3}}\right.$ and $\left.f_{1}^{\mathrm{STO}}\right)$ allows us to extract the effective dielectric constant of the $\mathrm{SrTiO}_{3}$ as follows:

$$
\varepsilon_{r, \text { eff }}^{\mathrm{STO}}=\varepsilon_{r, e f f}^{\mathrm{Al}_{2} \mathrm{O}_{3}}\left(f_{1}^{\mathrm{Al}_{2} \mathrm{O}_{3}} / f_{1}^{\mathrm{STO}}\right)^{2} .
$$

The effective dielectric constant accounts for the electricfield distribution through the vacuum above the substrate using a linear correction factor $\left(\varepsilon_{r, e f f}=\alpha \varepsilon_{r}\right)$. For dielectric constants in the range of the measured values for $\mathrm{SrTiO}_{3}\left(\varepsilon_{r}\right.$ $=2.000-30.000$ ), the correction factor $\alpha^{\mathrm{STO}}$ is calculated to be $\alpha_{\varepsilon_{\mathrm{r}}=2000}^{\mathrm{STO}}=0.5000$ and $\alpha_{\varepsilon_{\mathrm{r}}=30000}^{\mathrm{STO}}=0.4998$ (using analytical expressions from [21]). Since sapphire has a much lower dielectric constant which is also anisotropic, $\alpha^{\mathrm{Al}_{2} \mathrm{O}_{3}}$ is calculated using a finite elements simulation and is found to be $\alpha^{\mathrm{Al}_{2} \mathrm{O}_{3}}=$ 0.6004 (see Supplemental Material S2 [20]). The dielectric constant of $\mathrm{SrTiO}_{3}$ is strongly anisotropic, where the different crystal orientations show a variation of $\varepsilon_{\mathrm{r}}$ higher than a factor of 2 [7]. This means that planar geometries will sense an effective dielectric constant arising from an average over the different crystal orientations, domain configurations, and regions with different intensity of electric field (see Supplemental Material [20] S2).

A reflection measurement $\left|S_{11}\right|$ of the cavity modes is presented in Fig. 1(c), where 12 resonance modes are observed in the range from 1 to $300 \mathrm{MHz}$. The fundamental resonance frequency is $25.04 \mathrm{MHz}$, corresponding to $\varepsilon_{\mathrm{r}}=27400$, calculated from the observed frequency of the sapphire sample $f_{1}^{\mathrm{Al}_{2} \mathrm{O}_{3}}=1.241 \mathrm{GHz}$.

\section{ELECTRIC-FIELD DEPENDENCE OF $\varepsilon_{r}$}

The galvanic connection of the feedline to the CPW resonator enables us to apply a voltage difference between the central strip and the ground plane. The narrow $10-\mu \mathrm{m}$ gap converts a relatively small voltage to an intense and

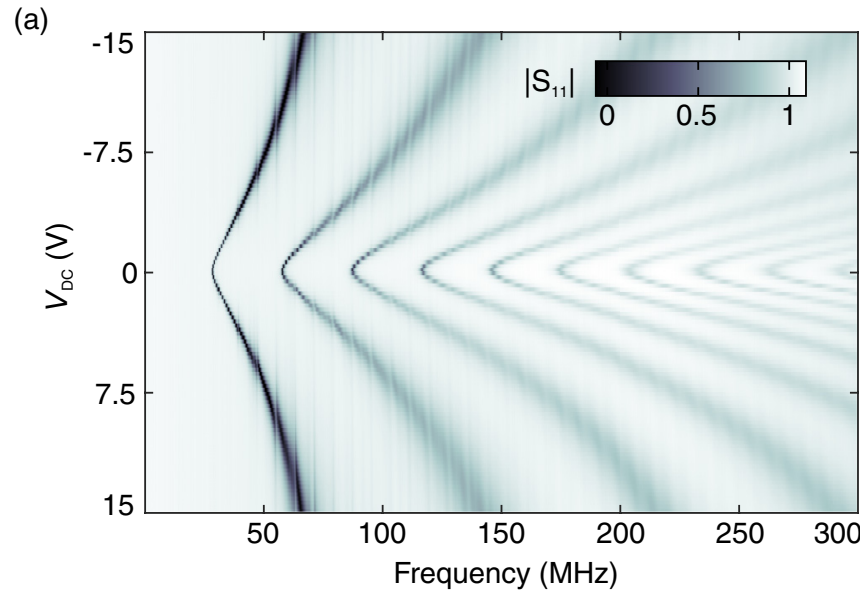

(b)



FIG. 2. dc voltage response of the resonator. (a) Color plot of $\left|\mathrm{S}_{11}\right|$, showing the frequency shift of the cavity modes upon applying $\mathrm{dc}$ voltage in the $\pm 15-\mathrm{V}$ range. (b) Voltage dependence of $\varepsilon_{r}$ of the $\mathrm{SrTiO}_{3}$ substrate calculated from the frequency shift of the first mode of the CPW resonator. The blue data points are measured right after cooldown, while the red data points are taken after subsequent voltage sweeps. The black lines are fits to the data using Eq. (2).

localized average electric field $(1 \mathrm{~V} \approx 100 \mathrm{kV} / \mathrm{m})$ whose magnitude is further enhanced in the proximity of the CPW by the inhomogeneous density of electric-field lines (see Supplemental Material S2 [20]). A similar local gating scheme was used recently in transport experiments in oxide systems [22-24]. The modification of the local dielectric constant caused by the dc bias results in a change of the total capacitance of the resonator and a corresponding shift of its resonance frequencies. By tracking the resonance frequency as a function of applied voltage across the gap, we probe the voltage dependence of the dielectric properties of $\mathrm{SrTiO}_{3}$ in a wide range of values.

In Fig. 2(a) we show a color plot of the reflection parameter $\left|\mathrm{S}_{11}\right|$ as a function of de voltage from -15 to $15 \mathrm{~V}$. By applying $-15 \mathrm{~V}$ to the central strip, the fundamental mode shifts from $25 \mathrm{MHz}$ to $66 \mathrm{MHz}$, which corresponds to a tunability of $160 \%$. By applying $30 \mathrm{~V}$ the fundamental frequency shifts to $90 \mathrm{MHz}$, resulting in a tunability of 260\% (see Supplemental Material S3 [20]). The extracted dielectric constant as a function of the applied voltage is plotted in Fig. 2(b). We 
calculated $\varepsilon_{r}^{\mathrm{STO}}$ during the first voltage sweep just after the cooldown (black dashed line) and after subsequent sweeps in the $\pm 15 \mathrm{~V}$ range (orange solid line). There is an evident nonreversible modification of the dielectric constant at low bias after the application of the voltage, where $\varepsilon_{r}^{\mathrm{STO}}(0)$ is reduced from the initial value of 27400 to less than 19000 . This behavior, typically observed in $\mathrm{SrTiO}_{3}$-based devices, is usually attributed to pinning of domain walls [25]. The electric-field dependence of the dielectric constant of $\mathrm{SrTiO}_{3}$ at low temperatures can be described in first approximation by the Landau-Ginzburg-Devonshire theory [26,27]:

$$
\varepsilon_{r}^{\mathrm{STO}}(E)=1+\frac{\varepsilon_{r}^{\mathrm{STO}}(0)}{\left[1+\left(E / E_{0}\right)^{2}\right]^{1 / 3}},
$$

where $E$ is the electric field, and $E_{0}$ is a parameter related to the tunability of the dielectric constant with electric field. During the first field sweep (black dashed line), we extracted a value for $E_{0}=71.5 \mathrm{kV} / \mathrm{m}$, whereas in subsequent sweeps (orange line), this value increased to $E_{0}=147 \mathrm{kV} / \mathrm{m}$, indicating a decrease in the polarizability of the $\mathrm{SrTiO}_{3}$. In Sec. V, we provide a detailed analysis of this effect. We note that both these values are about 1 order of magnitude lower than what was reported in literature from measurements on bulk single-crystal $\mathrm{SrTiO}_{3}$ with homogeneous electric fields [28]. This is due to the inhomogeneous distribution of the electric field, which is enhanced in the proximity of the central strip and the ground plane of the resonator. This allows us to affect the dielectric response at much lower voltages than those required in a double-capacitor geometry. It is worth mentioning, however, that despite the fact that we are probing the volume average of the properties of a strongly nonlinear material, the theoretical functional formulation still holds, supporting our analysis based on effective quantities.

\section{POWER AND FIELD DEPENDENCE OF MICROWAVE LOSSES}

Due to their low intrinsic losses [29], superconducting MoRe resonators provide a good platform for characterization of the loss mechanisms of the surrounding dielectric environment. Dielectric losses are typically evaluated through the loss tangent of the substrate $(\tan \delta)$, defined as the ratio between the imaginary $\left(\varepsilon^{\prime \prime}\right)$ and real part $\left(\varepsilon^{\prime}\right)$ of the relative permittivity,

$$
\tan \delta \equiv \varepsilon^{\prime \prime} / \varepsilon^{\prime},
$$

which can be directly obtained from fitting the reflection coefficient from a single port cavity $\left(\left|\mathrm{S}_{11}\right|\right)$ with its definition:

$$
\left|\mathrm{S}_{11}\right|=\frac{2 f_{0} / Q_{\text {int }}-\Delta f-4 i \pi\left(f-f_{0}\right)}{\Delta f-4 i \pi\left(f-f_{0}\right)},
$$

where $\Delta f$ is the extracted FWHM of the resonance peak, $Q_{\text {int }}$ is the internal quality factor, and $f_{0}$ its frequency. The loss tangent of the resonator $\left(1 / Q_{\text {int }}\right)$ usually incorporates both surface losses (originating from amorphous interfaces) and substrate losses. By comparison with the identical resonator fabricated on top of sapphire we can estimate the contribution of the radiation and quasiparticle losses to be less than $7 \times 10^{-4}$. We therefore use $1 / Q_{\text {int }}$ and $\tan \delta$ interchangeably.

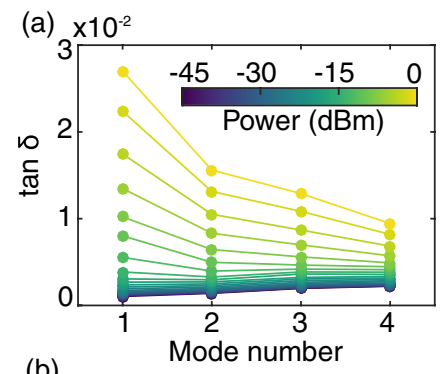

(c) $\times 10^{-2}$
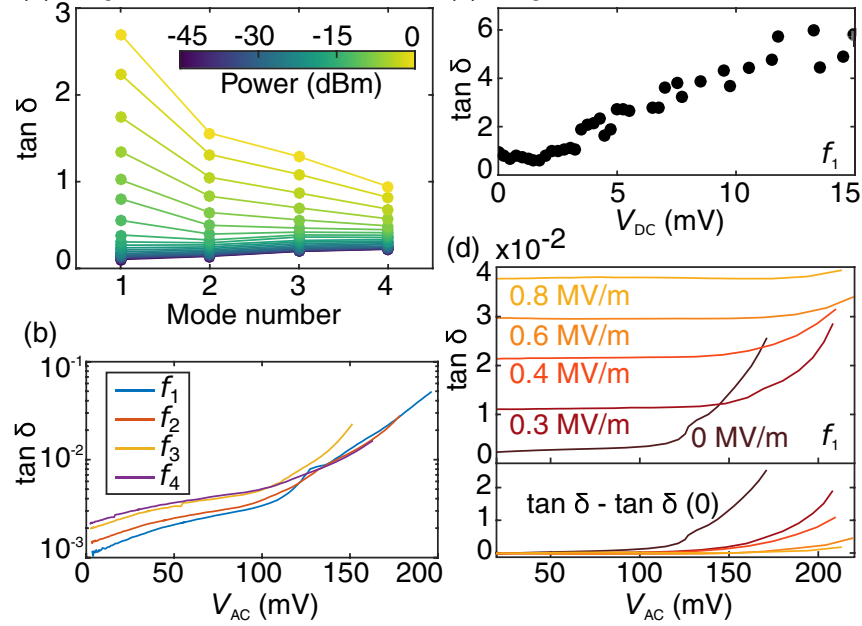

FIG. 3. Dielectric losses in $\mathrm{SrTiO}_{3}$ probed by the CPW resonator: (a) $\tan \delta$ vs mode number at zero dc field measured at different $\mathrm{rf}$ powers (curves are $2 \mathrm{dBm}$ apart). (b) $\tan \delta$ vs the ac voltage inside the cavity at resonance for the first four modes. (c) $\tan \delta$ vs dc voltage for the first mode of the resonator probed at fixed rf power. (d) Loss tangent for the first mode of the resonator as a function of the internal ac voltage for different values of the dc voltage. The bottom panel shows the overlapped curves, obtained by subtracting the value of $\tan \delta$ at minimum $V_{\mathrm{AC}}$.

Figure 3(a) shows the loss tangent for the first four resonance modes for different input rf powers $\left(P_{\text {in }}\right)$. Each curve corresponds to a different value of the rf power. For low values of $P_{\text {in }}\left(P_{\text {in }}<10 \mathrm{dBm}\right), \tan \delta$ for all modes is weakly affected by the input power and it increases with mode number. When increasing the input rf power, the losses become strongly affected by the ac field and their mode dependence is reversed, as lower modes seem to react more strongly to the input rf power.

In Fig. 3(b) we plot tan $\delta$ of the first four modes, but now as a function of the internal ac voltage rather than input power. This is important because the different internal quality factors of the modes result in different amplitudes of the ac voltage inside the resonator for the same input power. At low ac voltages $\left(V_{\mathrm{AC}}<\right.$ $90 \mathrm{mV}$ ), we observe a monotonic dependence of $\tan \delta$ on mode number: lower modes exhibit lower $\tan \delta$. This suggests that $\tan \delta$ is frequency dependent, similar to what was observed in [10] for gigahertz frequencies. In this range, all modes show a linear dependence on the ac voltage with similar slopes (see Supplemental Material S4 [20]). For higher amplitudes of the ac voltage $\left(V_{\mathrm{AC}}>90 \mathrm{mV}\right)$ we find an exponential increase of the losses for all four modes. In the high-power region, the peaks become progressively broader and we also see signatures of nonlinear damping. Details on the derivations and fits of the data are presented in Supplemental Material S4 [20].

The observed power dependence of $\tan \delta$ is not consistent with the typical behavior of dissipation in the two-level system (TLS) model, a common source of losses at the metal-substrate/metal-air interfaces, since such interfacial losses usually show an opposite saturation behavior [30,31]. This suggests that the measured $\tan \delta$ likely originates from substrate losses. 
To better understand the loss mechanisms, in Fig. 3(c) we also investigate the dependence of $\tan \delta$ as a function of $\mathrm{dc}$ electric field extracted from the resonance measurements for the first dc sweep. The increase of $\tan \delta$ with $V_{\mathrm{DC}}$ is in agreement with the so-called quasi-Debye mechanism $[32,33]$, which is the dominant dissipation channel in incipient ferroelectrics. This mechanism originates from the interplay between dc and ac components of the excitation. The dc field breaks the lattice symmetry, making the phonon spectrum field-dependent. The ac field acts as a time modulation of the phonon frequencies, which are continuously driven out of equilibrium with consequent energy dissipation. This increase has been already observed in $\mathrm{SrTiO}_{3}$ single crystals [34], while it is in striking difference with several studies on thin films, where the application of an electric field leads to a decrease in $\tan \delta[14,35]$.

Figure 3(d) shows the ac-induced losses for different values of the dc voltage for the first resonance mode where, similarly to Figs. 3(a) and 3(b), two different regimes can be distinguished. It is possible to compare the losses related to the ac field at different dc bias by considering their rescaled values: $\tan \delta\left(V_{\mathrm{AC}}\right)-\tan \delta\left(V_{\mathrm{AC}}=0\right)$, which are plotted in the bottom panel of Fig. 3(d). The presence of a dc field reduces the magnitude of the ac-induced losses, which suggests that the two dissipation channels are not fully independent.

In contrast to what is observed in thin films, we measured an increase of the losses with the magnitude of both ac and dc fields. According to Zubko and Vasil'ev [36], the ac and dc field dependence of the losses is determined to a large extent by the parameter $\xi_{\mathrm{S}}$, which characterizes the quality of the crystal $\left(\xi_{\mathrm{S}} \approx 0.02-0.06\right.$ for high-quality single-crystal $\mathrm{SrTiO}_{3}$ and $\xi_{\mathrm{S}}>0.5$ for thin films $\left.[10,37]\right)$. For values of $\xi_{\mathrm{S}}<0.25, \tan \delta$ should monotonically increase with both dc and ac electric fields, which is in agreement with our measurements.

\section{DEPENDENCE OF $\varepsilon_{r}$ AND $\tan \delta$ ON FIELD POLARITY}

In Fig. 4, we explore the influence of voltage polarity on the hysteretic response of the dielectric properties of $\mathrm{SrTiO}_{3}$. To do so, we followed the measurement sequences sketched in Fig. 4(a): starting from zero bias, we applied a voltage ramp up to a target value $V_{\max }$ and then back to $V=0$, where the $\mathrm{CPW}$ resonator spectrum is acquired. This procedure is repeated for increasing values of $V_{\max }$ up to $15 \mathrm{~V}$, always with the same polarity. We then perform a thermal cycle up to room temperature, while keeping the line grounded, to restore the initial sample conditions. We probed under three biasing schemes: positive, negative, and positive after negative (zero average).

Figure 4(b) shows that the value of $\varepsilon_{r}$ at zero field is not symmetric with respect to the applied field polarity and, in particular, it is significantly affected only by negative biases. We thus link the origin of the reduction of permittivity to the presence of oxygen vacancies, since a negative bias can increase their concentration in the surroundings of the waveguide, where the dielectric environment is probed. This scenario is supported by the partial recovery observed when a positive voltage follows a negative one (yellow squares of
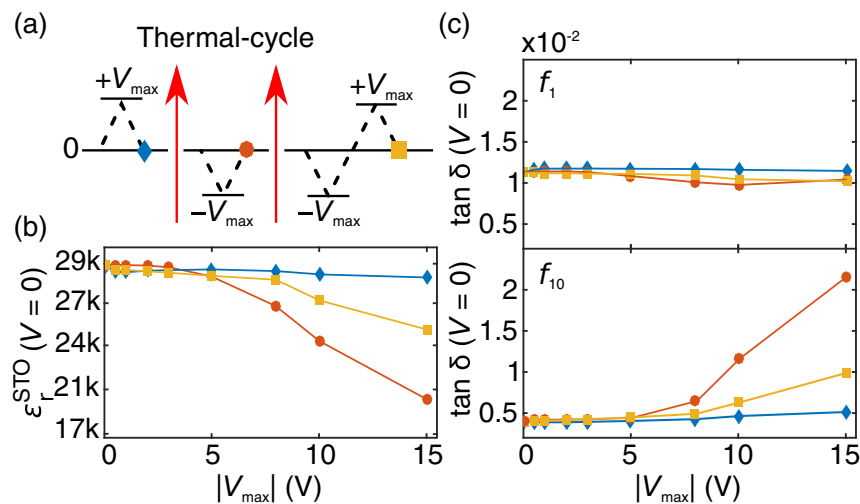

FIG. 4. Hysteretic effects in the dielectric response of $\mathrm{SrTiO}_{3}$. (a) Diagram showing the biasing and measurement sequence used to characterize the dependence of $\varepsilon_{r}^{\mathrm{STO}}$ (b) and $\tan \delta$ (c) on the field polarity. Measurements are acquired at zero dc bias after the application of a given voltage difference $\left(V_{\max }\right)$ between the line and the ground plane. We kept a constant polarity for the different $V_{\max }$ values. After reaching $V_{\max }= \pm 15 \mathrm{~V}$, a thermal cycle to $300 \mathrm{~K}$ restores the initial conditions of the sample. (Top: fundamental mode $\left(f_{1}\right)$, bottom: mode $f_{10}$.)

Fig. 4). This explains the low tunability reported for CPW resonators realized on top of $\mathrm{SrTiO}_{3}$ thin films, since even the very low defects concentration present at the surface of our substrate is already capable of lowering the dielectric response by more than $25 \%$ after the application of $15 \mathrm{~V}$. Even though the cooldown conditions were kept unchanged, the values of $\epsilon_{r}$ and $\tan \delta$ at zero bias upon cooldown show a spread of about $2 \%$. This value is negligible with respect to the observed variations and may be caused by different stable configurations of the oxygen vacancies at room temperature. This asymmetric response is also observed in the dielectric losses presented in Fig. 4(c). However, we note that the measured response depends unexpectedly on the mode number. As a general trend, a monotonic increase of the losses is observed, which is explained by a progressive accumulation of polarized defects for negative biases [33]. However, for low mode numbers and bias values below $10 \mathrm{~V}$, the application of an electric field results in a decrease of $\tan \delta$. In the Supplemental Material S5 and S6 [20], we provide an extensive mapping of the losses showing the evolution of $\tan \delta$ as a function of the maximum applied voltage, mode number, and input power. In particular, upon having applied a dc voltage of $-15 \mathrm{~V}$ and performing the same analysis as in Fig. 3(a), we observe a stronger dependence of $\tan \delta$ on mode number at low powers, whereas, for higher input powers, $\tan \delta$ of the first four modes shows a similar behavior.

We also extract the polarizability parameter $E_{0}$ and the horizontal shift of the maximum of $\varepsilon_{r}^{\text {STO }}$ by fitting the $\varepsilon_{r}^{\text {STO }}$ vs $V_{\text {DC }}$ curves during the voltage sweeps (Supplemental Material S7 [20]). Finally, to support our interpretation of the fatigue mechanism, in Fig. S8 of the Supplemental Material we perform a measurement of the time evolution of $\varepsilon_{r}^{\text {STO }}$ upon applying a small negative dc voltage [20]. The time response of the dielectric constant indicates the presence of slow relaxation dynamics, which is in accordance with the proposed model of drift of charged defects. 
An advantage of the described method is that the superconducting cavity is fabricated directly on top of the $\mathrm{SrTiO}_{3}$ and serves as a local probe of the underlying dielectric. In addition, the use of a reference sample facilitates the extraction of quantitative information on the dielectric properties of the material. This measurement technique is compatible with a wide variety of complex oxides, it enables local electrostatic gating, and it can serve as a platform for time-resolved measurements in the millisecond range.

\section{CONCLUSIONS}

In conclusion, we realized a superconducting coplanar waveguide resonator directly on top of a single-crystal $\mathrm{SrTiO}_{3}(001)$ substrate and probed its dielectric properties as a function of dc voltage, rf power injected in the line, and mode number. The effective dielectric constant measured in the initial condition was $\sim 27000$, lowered below 4000 by applying a dc voltage of $15 \mathrm{~V}$. The inhomogeneous distribution of the electric field in the resonator geometry allowed us to uncover an asymmetric response with respect to the field polarity of both $\varepsilon_{r}$ and $\tan \delta$, with a progressive reduction of the resonator's tunability range, pointing towards the presence of charged defects, such as oxygen vacancies, as the origin of the observed behavior. The narrow gap $(10 \mu \mathrm{m})$ increases the sensitivity of the microwave response to effects on a length scale of the order of the gap size. By further shrinking its dimensions, a microwave cavity can act as a local probe capable of studying, for example, the dynamics and the dielectric behavior of single domain walls, characteristic of $\mathrm{SrTiO}_{3}$ at low temperatures. Our results demonstrate the sensitivity of superconducting microwave cavities as probes of quantum matter and provide a robust background for future studies on oxide heterostructures probed by superconducting resonators.

\section{ACKNOWLEDGMENTS}

The authors would like to thank Daniel Bothner and Mark Jenkins Sanchez for their help during data analysis, and Peter G. Steeneken and Teun M. Klapwijk for the fruitful discussions. This work was supported by The Netherlands Organisation for Scientific Research (NWO/OCW) as part of the Frontiers of Nanoscience program and by the Dutch Foundation for Fundamental Research on Matter (FOM). The research leading to these results has received funding from the European Research Council under the European Union's H2020 programme/ERC Grant Agreement No. 677458.

D.D. and N.M. contributed equally to this work.
[1] A. Wallraff, D. I. Schuster, A. Blais, L. Frunzio, R.-S. Huang, J. Majer, S. Kumar, S. M. Girvin, and R. J. Schoelkopf, Strong coupling of a single photon to a superconducting qubit using circuit quantum electrodynamics, Nature (London) 431, 162 (2004).

[2] D. I. Schuster, A. P. Sears, E. Ginossar, L. DiCarlo, L. Frunzio, J. J. L. Morton, H. Wu, G. A. D. Briggs, B. B. Buckley, D. D. Awschalom, and R. J. Schoelkopf, High-Cooperativity Coupling of Electron-Spin Ensembles to Superconducting Cavities, Phys. Rev. Lett. 105, 140501 (2010).

[3] H. Huebl, C. W. Zollitsch, J. Lotze, F. Hocke, M. Greifenstein, A. Marx, R. Gross, and S. T. B. Goennenwein, High Cooperativity in Coupled Microwave Resonator Ferrimagnetic Insulator Hybrids, Phys. Rev. Lett. 111, 127003 (2013).

[4] N. Reyren, S. Thiel, A. D. Caviglia, L. F. Kourkoutis, G. Hammerl, C. Richter, C. W. Schneider, T. Kopp, A.-S. Rüetschi, D. Jaccard, M. Gabay, D. A. Muller, J.-M. Triscone, and J. Mannhart, Superconducting interfaces between insulating oxides, Science 317, 1196 (2007).

[5] S. Farokhipoor, C. Magén, S. Venkatesan, J. Íñiguez, C. J. Daumont, D. Rubi, E. Snoeck, M. Mostovoy, C. D. Graaf, A. Müller et al., Artificial chemical and magnetic structure at the domain walls of an epitaxial oxide, Nature (London) 515, 379 (2014).

[6] P. Zubko, J. C. Wojdeł, M. Hadjimichael, S. Fernandez-Pena, A. Sené, I. Lukýanchuk, J.-M. Triscone, and J. Íñiguez, Negative capacitance in multidomain ferroelectric superlattices, Nature (London) 534, 524 (2016).

[7] T. Sakudo and H. Unoki, Dielectric Properties of $\mathrm{SrTiO}_{3}$ at Low Temperatures, Phys. Rev. Lett. 26, 851 (1971).

[8] R. C. Neville, B. Hoeneisen, and C. A. Mead, Permittivity of strontium titanate, J. Appl. Phys. 43, 2124 (1972).
[9] J. Krupka, R. G. Geyer, M. Kuhn, and J. H. Hinken, Dielectric properties of single crystals of $\mathrm{Al}_{2} \mathrm{O}_{3}, \mathrm{LaAlO}_{3}, \mathrm{NdGaO}_{3}$, $\mathrm{SrTiO}_{3}$, and $\mathrm{MgO}$ at cryogenic temperatures, IEEE Trans. Microwave Theory Tech. 42, 1886 (1994).

[10] R. G. Geyer, B. Riddle, J. Krupka, and L. A. Boatner, Microwave dielectric properties of single-crystal quantum paraelectrics $\mathrm{KTaO}_{3}$ and $\mathrm{SrTiO}_{3}$ at cryogenic temperatures, J. Appl. Phys. 97, 104111 (2005).

[11] J. Hemberger, P. Lunkenheimer, R. Viana, R. Böhmer, and A. Loidl, Electric-field-dependent dielectric constant and nonlinear susceptibility in $\mathrm{SrTiO}_{3}$, Phys. Rev. B 52, 13159 (1995).

[12] A. Antons, J. B. Neaton, K. M. Rabe, and D. Vanderbilt, Tunability of the dielectric response of epitaxially strained $\mathrm{SrTiO}_{3}$ from first principles, Phys. Rev. B 71, 024102 (2005).

[13] D. Galt, J. C. Price, J. A. Beall, and R. H. Ono, Characterization of a tunable thin film microwave $\mathrm{YBa}_{2} \mathrm{Cu}_{3} \mathrm{O}_{7-\mathrm{x}} / \mathrm{SrTiO}_{3}$ coplanar capacitor, Appl. Phys. Lett. 63, 3078 (1993).

[14] A. T. Findikoglu, Q. X. Jia, I. H. Campbell, X. D. Wu, D. Reagor, C. B. Mombourquette, and D. McMurry, Electrically tunable coplanar transmission line resonators using $\mathrm{YBa}_{2} \mathrm{Cu}_{3} \mathrm{O}_{7-\mathrm{x}} / \mathrm{SrTiO}_{3}$ bilayers, Appl. Phys. Lett. 66, 3674 (1995).

[15] A. T. Findikoglu, Q. X. Jia, X. D. Wu, G. J. Chen, T. Venkatesan, and D. W. Reagor, Tunable and adaptive bandpass filter using a nonlinear dielectric thin film of $\mathrm{SrTiO}_{3}$, Appl. Phys. Lett. 68, 1651 (1996).

[16] J. Sok, J. S. Lee, and E. H. Lee, Tunability of resonance frequencies in a superconducting microwave resonator by using $\mathrm{SrTiO}_{3}$ ferroelectric films, J. Korean Phys. Soc. 33, 158 (1998). 
[17] H. Fuke, Y. Terashima, H. Kayano, and H. Yoshino, Electrically tunable $\mathrm{YBa}_{2} \mathrm{Cu}_{3} \mathrm{O}_{\mathrm{y}}$ resonators using interdigital electrodes and dielectric film, Phys. C Supercond. 336, 80 (2000).

[18] M. Adam, D. Fuchs, and R. Schneider, $\mathrm{YBa}_{2} \mathrm{Cu}_{3} \mathrm{O}_{7}$ microwave resonator tuned by epitaxial $\mathrm{SrTiO}_{3}$ thin films, Phys. C Supercond. 372-376, 504 (2002).

[19] D. G. Blair and I. N. Evans, High-Q microwave properties of a sapphire ring resonator, J. Phys. D. Appl. Phys. 15, 1651 (1982).

[20] See Supplemental Material at http://link.aps.org/supplemental/ 10.1103/PhysRevB.95.214513 for additional data on the sapphire sample, FEM simulations, dc frequency tunability, calculations of the ac voltage inside the cavity at resonance, fits of the frequency responses, hysteretic effects in $\tan \delta$ for all modes, the effect of bias polarity on the polarizability of the STO and a time evolution of the dielectric constant of STO upon applying a dc voltage.

[21] R. N. Simons, Coplanar Waveguide Circuits, Components, and Systems (Wiley, Cleveland, OH, 2004).

[22] D. Stornaiuolo, S. Gariglio, A. Fête, M. Gabay, D. Li, D. Massarotti, and J.-M. Triscone, Weak localization and spin-orbit interaction in side-gate field effect devices at the $\mathrm{LaAlO}_{3} / \mathrm{SrTiO}_{3}$ interface, Phys. Rev. B 90, 235426 (2014).

[23] W. Liu, S. Gariglio, A. Fête, D. Li, M. Boselli, D. Stornaiuolo, and J.-M. Triscone, Magneto-transport study of top- and backgated $\mathrm{LaAlO}_{3} / \mathrm{SrTiO}_{3}$ heterostructures, APL Mater. 3, 062805 (2015).

[24] A. M. R. V. L. Monteiro, D. J. Groenendijk, N. Manca, E. Mulazimoglu, S. Goswami, Y. Blanter, L. M. K. Vandersypen, and A. D. Caviglia, Side gate tunable Josephson junctions at the $\mathrm{LaAlO}_{3} / \mathrm{SrTiO}_{3}$ interface, Nano Lett. 17, 715 (2017).

[25] M. Honig, J. A. Sulpizio, J. Drori, A. Joshua, E. Zeldov, and S. Ilani, Local electrostatic imaging of striped domain order in $\mathrm{LaAlO}_{3} / \mathrm{SrTiO}_{3}$, Nat. Mater. 12, 1112 (2013).

[26] L. Landau, L. Pitaevskii, and E. Lifshitz, Electrodynamics of Continuous Media, 2nd ed. (Pergamon Press, Oxford, 1984).

[27] C. Ang and Z. Yu, DC electric-field dependence of the dielectric constant in polar dielectrics: Multipolarization mechanism model, Phys. Rev. B 69, 174109 (2004).

[28] O. G. Vendik, E. K. Hollmann, A. B. Kozyrev, and A. M. Prudan, Ferroelectric tuning of planar and bulk microwave devices, J. Supercond. 12, 325 (1999).
[29] V. Singh, B. H. Schneider, S. J. Bosman, E. P. J. Merkx, and G. A. Steele, Molybdenum-rhenium alloy based high-Q superconducting microwave resonators, Appl. Phys. Lett. 105, 222601 (2014).

[30] J. Gao, M. Daal, A. Vayonakis, S. Kumar, J. Zmuidzinas, B. Sadoulet, B. A. Mazin, P. K. Day, and H. G. Leduc, Experimental evidence for a surface distribution of two-level systems in superconducting lithographed microwave resonators, Appl. Phys. Lett. 92, 152505 (2008).

[31] J. Wenner, R. Barends, R. C. Bialczak, Y. Chen, J. Kelly, E. Lucero, M. Mariantoni, A. Megrant, P. J. J. O’Malley, D. Sank, A. Vainsencher, H. Wang, T. C. White, Y. Yin, J. Zhao, A. N. Cleland, and J. M. Martinis, Surface loss simulations of superconducting coplanar waveguide resonators, Appl. Phys. Lett. 99, 113513 (2011).

[32] A. Tagantsev, DC-electric-field-induced microwave loss in ferroelectrics and intrinsic limitation for the quality factor of a tunable component, Appl. Phys. Lett. 76, 1182 (2000).

[33] O. G. Vendik, S. P. Zubko, and M. A. Nikol'ski, Microwave loss-factor of $\mathrm{Ba}_{\mathrm{x}} \mathrm{Sr}_{1-\mathrm{x}} \mathrm{TiO}_{3}$ as a function of temperature, biasing field, barium concentration, and frequency, J. Appl. Phys. 92, 7448 (2002).

[34] A. T. Findikoglu, D. W. Reagor, K. O. Rasmussen, A. R. Bishop, N. Groİ,nbech-Jensen, Q. X. Jia, Y. Fan, C. Kwon, and L. A. Ostrovsky, Electrodynamic properties of coplanar waveguides made from high-temperature superconducting $\mathrm{YBa}_{2} \mathrm{Cu}_{3} \mathrm{O}_{7-\delta}$ electrodes on nonlinear dielectric $\mathrm{SrTiO}_{3}$ substrates, J. Appl. Phys. 86, 1558 (1999).

[35] A. T. Findikoglu, Q. X. Jia, C. Kwon, D. W. Reagor, G. Kaduchak, K. Rasmussen, and A. R. Bishop, Comparative study of broadband electrodynamic properties of single-crystal and thin-film strontium titanate, Appl. Phys. Lett. 75, 4189 (1999).

[36] S. P. Zubko and A. N. Vasil'ev, A model for the dependence of the dielectric loss factor of a ferroelectric on the microwave signal amplitude, Phys. Solid State 51, 1545 (2009).

[37] O. G. Vendik and S. P. Zubko, Modeling the dielectric response of incipient ferroelectrics, J. Appl. Phys. 82, 4475 (1997). 